\title{
Modelo de filas para análise de redes de múltiplos saltos
}

\section{Row model for multi-hop network analysis}

DOI: $10.46814 / \operatorname{lajdv} 4$ n1-001

Recebimento dos originais: 01/12/2021

Aceitação para publicação: 03/01/2022

\section{Cecília de Azevedo Castro César}

Instituto Tecnológico da Aeronáutica

ITA Praça Marechal Eduardo Gomes, 50 Vila das Acácias - São José dos Campos / SP

E-mail: Brasilcecilia@ita.br

\section{Solon Venâncio de Carvalho}

Instituto Nacional de Pesquisas Espaciais - INPE, São Paulo, Brasil. Av. dos Astronautas, 1758 - Jd. Da Granja - São José dos Campos / SP

E-mail: BrasilSolon@lac.inpe.br

\section{RESUMO}

Neste artigo é apresentado um Modelo Analítico de Filas para auxiliar na decisão de quais dados, em uma malha de distribuição, um nó deve priorizar no atendimento: dados vindos de nós vizinhos, ou dados gerados localmente. Esta decisão visa melhorar a vazão da rede como um todo e diminuir o atraso. O projeto de uma Rede de Computadores sem Fio em Malha é uma possível aplicação deste modelo, pois sua topologia envolve o encaminhamento de pacotes de dados passando por diversos nós intermediários situados entre a origem e o destino. A análise de desempenho oferecida pelo modelo pode auxiliar o projeto da rede em relação à topologia, à carga submetida e aos serviços a serem implantados. Propõe-se também um critério de equidade que minimize o prejuízo dos nós mais distantes sem, contudo, prejudicar a vazão total. Os testes realizados indicam ganhos significativos na aplicação do critério de equidade.

Palavras chave: Modelo de Filas, Redes de Computadores sem Fio em Malha, Análise de Desempenho.

\begin{abstract}
This paper presents a Queuing Analytical Model to assist in deciding which data in a distribution network, a node must prioritize in routing: data from the neighboring nodes or data generated locally. This decision is aimed at improving the throughput of the network as a whole and reducing the delay. The design of a Wireless Mesh Network (WMN) is a good application of this model, since its topology involves the routing of data packets passing through several intermediate nodes located between the source and destination. The performance analysis provided by the model can assist the project in relation to the network topology, the imposed load and the services to be deployed. It is proposed a fairness criterion that minimizes the damage of more distant nodes without, however, affect the total flow. The tests indicated significant gains in applying the criterion of fairness.
\end{abstract}

Keywords: Queuing Model, Wireless Mesh Networks, Performance Analysis. 


\section{INTRODUÇÃO}

Em diversos cenários de decisão, há a situação na qual os nós em uma malha de distribuição estão conectados a outros nós para realizar o encaminhamento do objeto alvo. Um cenário típico são as Redes de Computadores sem Fio em Malha (Wireless Mesh Network - WMN) cujo objeto alvo são os pacotes de dados. Estas redes têm a característica de que cada nó pode estar conectado a outros nós e atuar como hospedeiro ou roteador para seus vizinhos, encaminhando pacotes para nós que não estão no alcance direto de seu destino (Zhang et al., 2006).

A Figura 1 ilustra uma WMN - um exemplo de redes de múltiplos saltos - que tem recebido muita atenção pela facilidade de instalação, grande cobertura e robustez, pois em caso de falha em um enlace, o encaminhamento pode continuar por caminhos alternativos (Akyildiz et al., 2005).

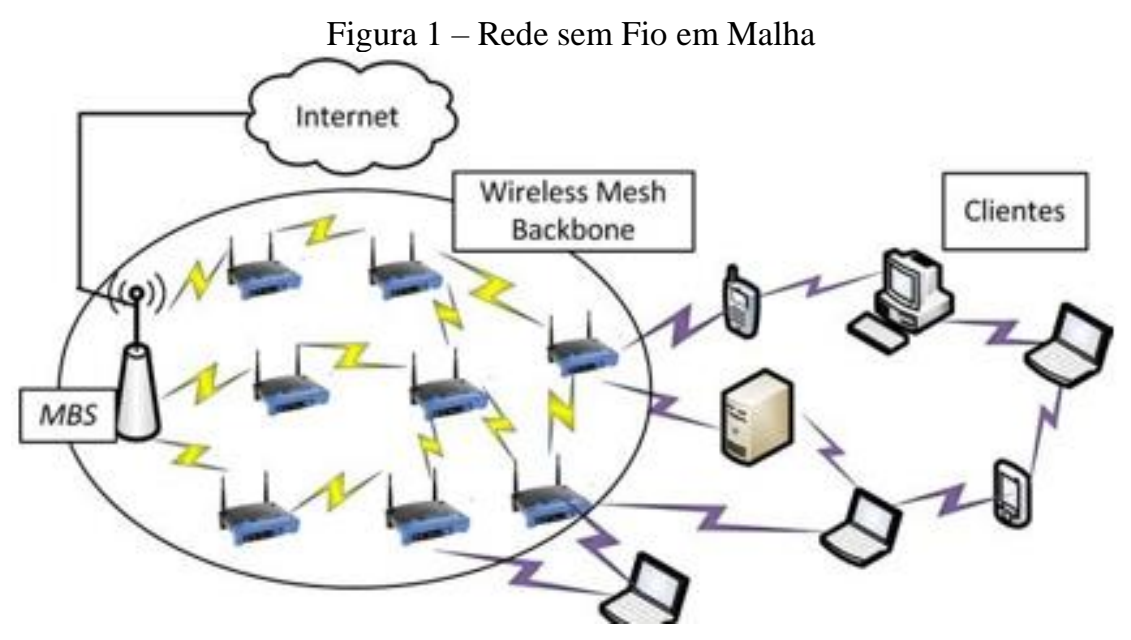

$\mathrm{Na}$ Figura 1 mostra-se um backbone em malha com roteadores que atuam como encaminhadores, e que também podem estar ligados diretamente a clientes que fazem suas solicitações. Os pacotes caminham pela malha até chegar a roteadores especiais de grandecapacidade, conectados à Internet, chamados de MBS (Mesh Base Station), que fazem o papel de gateway. Os clientes também podem atuar como roteadores para seus vizinhos. Em cada nó, a decisão a tomar é: priorizar pacotes gerados localmente ou pacotes dos vizinhos para os quais onó corrente é encaminhador.

Os protocolos recentemente desenvolvidos IEEE 802.11s (Zhang et al., 2006) e IEEE 802.16 (IEEE 802.16-2004,2004) incluem especificações para construir uma rede em malha, admitindo novos clientes a qualquer momento. O IEEE 802.16 também especifica a comunicaçãocentralizada, com as decisões tomadas pela MBS, ou descentralizada, onde cada nó toma sua própria decisão quanto ao envio de pacotes. Enquanto alguns trabalhos modelaram as redesIEEE 802.11s e as redes IEEE 802.16 (Cesar, 2011), alguns esforços foram feitos para obter modelos gerais em redes de múltiplos saltos que fossem independentes de protocolos específicos (Gupta e Kumar, 2000), (Liu et al., 2003). 
Para determinar o atraso fim-a-fim, é preciso considerar o atraso em cada nó intermediário, onde eventualmente os pacotes devem aguardar o encaminhamento em uma fila. Por esta razão, Teoria de filas mostra-se muito adequada no tratamento deste problema. Alguns modelos usam Teoria de filas para investigar o efeito da localização do nó na análise de desempenho da rede. O modelo de Liu e Liao (2008) fornece medidas de desempenho de acordo com a variação da distância da estação à MBS, modelando uma estação como uma fila. Min et al.(2009) aplicam uma formulação semelhante, porém com o objetivo de interconectar redes locais sem fio (WLAN-Wireless Local Area Network) através de uma WMN. Kuo e Liao (2007) modelaram a distribuição de probabilidade do número esperado de saltos na entrega de pacotes numa rede de densidade arbitrária.

Um problema comum em redes de múltiplos saltos, enfatizado por estes modelos, é que a topologia tem grande impacto no desempenho, pois, redes contendo nós muito distantes do gateway principal, ou muito densas, podem resultar em vazão e atraso insuficientes para certotipo de serviço como tráfego multimídia. Este problema justifica a adoção de critérios de equidade, ou seja, é fundamental garantir a divisão equânime de recursos sem prejudicar a eficiência total do sistema. Liu e Liao (2008) aplicaram o critério max-min (Gambiroza, 2004), dividindo a banda disponível igualmente entre todos os nós de um determinado fluxo. Duffy et al.(2006) de forma semelhante determinam que uma estação ganhará tantas oportunidades detransmissão quanto o número de estações clientes de que trata. Wang et al. (2008) compartilhamo tempo de uso do canal, justificando que isto é ainda mais justo que o compartilhamento de banda.

O modelo foco deste trabalho procura apoiar a decisão de projeto de uma rede em malha, oferecendo subsídios para limitar o tamanho da rede em termos do número de saltos ouem termos de sua densidade, dependendo dos resultados dos parâmetros de qualidade observados. Os parâmetros analisados, neste trabalho, são a vazão e o atraso fim-a-fim na malha. A principal contribuição do trabalho é um modelo de fila para análise do desempenho de uma rede de múltiplos saltos. Uma segunda contribuição é a proposta de um critério de equidade para distribuição da banda que traz melhorias significativas nos parâmetros de análise da rede.

O restante do artigo está organizado da seguinte maneira: na Seção II, o modelo do sistema é derivado, chegando às fórmulas para cálculo de vazão e atraso; na Seção III, aplica-se ao modelo um critério de equidade concedendo uma fatia justa da banda a cada nó; na Seção IV, são apresentados os resultados comparativos da aplicação do modelo a diferentes topologias de rede; e, por fim, a conclusão na Seção V. 


\section{MODELO DO SISTEMA}

O modelo em questão considera que dentro de um nó podem existir duas filas do tipo $\mathrm{M} / \mathrm{M} / 1 / \mathrm{K}$ (Gross e Harris, 1998): $Q_{s}$, uma fila contendo os dados gerados no próprio nó, e $Q_{r}$ com os dados encaminhados pelo nó advindos de seus vizinhos que estão a um salto a mais de distância da MBS. Considera-se aqui que ambas as filas podem estar vazias ou estar bloqueadas pelo excesso de pacotes, ao contrário de Liu e Liao (2008) que assumem que a fila $Q_{s}$ é acumulada (backlogged), ou seja, nunca está vazia nem excessivamente cheia e, portanto, não é do tipo $\mathrm{M} / \mathrm{M} / 1 / \mathrm{K}$.

Considera-se que ambas as filas tem processo de entrada Poisson com taxas $\lambda_{s}$ e $\lambda_{r}$, respectivamente.

O cenário considerado é uma rede de múltiplos saltos estática com $N$ nós em malha que tem um gateway que serve como o sorvedouro (sink) da rede. Por se tratar de um modelo geral não vinculado a um protocolo específico, assume-se que há uma probabilidade $p(x)$ de um nó, a $x$ saltos de distância do gateway, ganhar o acesso ao meio. Esta probabilidade representa sucintamente a estratégia de controle de acesso ao meio (MAC-Medium Access Control).

Quando o nó tem a oportunidade de transmissão, havendo duas filas para sua escolha,há a probabilidade $q(x)$ de a fila de encaminhamento $Q_{r}$ ser a escolhida, e a probabilidade $1-q(x)$ de a fila local $Q_{s}$ ser a escolhida para transmitir seu pacote, como ilustrado na Figura 2.

Seja $N(x)$ o número esperado de nós a $x$ saltos do gateway e $N_{r}(x)$ o número esperado denós a $(x+1)$ saltos para os quais o nó $x$ encaminhará pacotes:

$$
N_{r}(x)=N(x+1) / N(x)
$$

A taxa de serviço dos pacotes no nó a $x$ saltos, $\mu(x)$, é o tempo entre uma transmissão e outra, ou o tempo necessário para obter novo acesso ao canal.

O tempo entre uma transmissão e outra depende de $p(x)$ e da duração das fatias de tempo (slot) que são disputadas no quadro, considerando um sistema baseado na transmissão TDMA (TimeDivision Multiple-Access). A Figura 3 ilustra o tempo entre duas transmissões, $T_{i}(x)$, e a duração de uma fatia de tempo, $t_{c}$.

Figura 2: Nó com duas filas internas

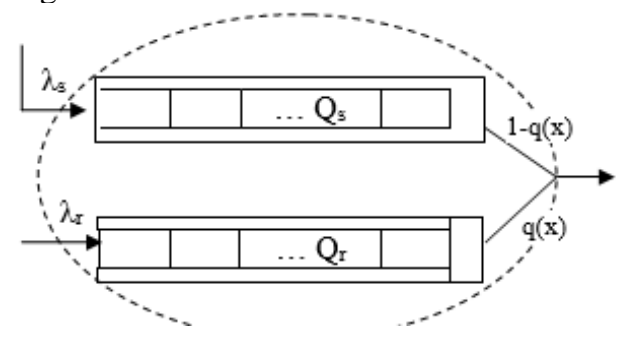

Figura 3: Tempo entre duas transmissões

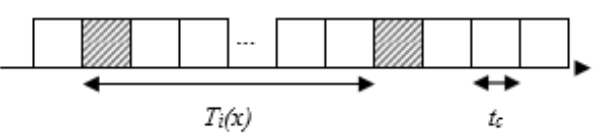

Figura 3: Tempo entre duas transmissões 
A probabilidade de o nó não conseguir a transmissão até atingir $m$ fatias de tempo,equivale a uma probabilidade binomial de ter zero sucesso em m tentativas:

$$
P(X=0)=\frac{m !}{0 !(m-0) !} p(x)^{0} .\left(1-p(x)^{)^{m}},\right.
$$

ou seja, $P\left(T_{i}(x)>m . t c\right)=(1-p(x))^{m}$.

Por outro lado, pode-se considerar que o processo de chegada de oportunidades de transmissão é um processo Poisson, ou seja, aproxima-se a variável aleatória $T_{i}(x)$ como variável aleatória exponencial com média $1 / \mu(x) e$

$$
P\left(T_{i}(x) \square m \cdot t c\right)=1-e^{-\square(x) \cdot m \cdot t c} .
$$

A probabilidade binomial pode ser aproximada por probabilidade de Poisson quando o número de tentativas $m$ é grande e a probabilidade de sucesso $p(x)$ é pequena. Com esta aproximação tem-se que $(1-p(x))^{m} \square e^{-\square(x) \cdot m \cdot t c}$ de onde se extrai $\mu(x)$ :

$$
\mu(x)=-\ln (1-p(x)) / t c
$$

e ainda se $p(x)$ for pequeno, pode-se aproximar $-\ln (1-p(x)) \sim p(x)$ e assim

$$
\mu(x)=p(x) / t c .
$$

Cada uma das filas vai contribuir para taxa de serviço do nó se for escolhida paratransmitir, ou seja,

$$
\begin{aligned}
& \mu_{r}(x)=\mu(x) q(x), \\
& \mu_{s}(x)=\mu(x)(1-q(x)) .
\end{aligned}
$$

É preciso considerar que a fila escolhida pode estar vazia. Assim, a taxa efetiva de saídade cada fila é a taxa de serviço vezes a probabilidade de a fila não estar vazia:

$$
\begin{aligned}
& \square_{s}(x)=\mu_{s}(x)\left[1-P_{s o}(x)\right] e \\
& \square_{r}(x)=\mu_{r}(x)\left[1-P_{r o}(x)\right],
\end{aligned}
$$


e a taxa de saída do nó $x$ é a soma da taxa de saída de cada fila:

$$
\square(x)=\square_{s}(x)+\square_{r}(x)
$$

Supõe-se que o processo de chegada à fila $Q_{s}$ é exponencial com média $1 / \square_{s}(x)$ e os tempos de serviço também são exponencialmente distribuídos com média $1 / \mu_{s}(x)$. Com estas suposições pode-se adotar o modelo de fila M/M/1/K para a fila $Q_{s}$ (Gross e Harris, 1998). A fila $Q_{r}$ em $x$ é alimentada pelos nós a $x+1$ saltos e, como se supôs que $T_{i}(x)$ obedece a processo de Poisson, a sobreposição destes processos independentes é ainda um processo de Poisson. Desta maneira, pode se supor que a fila $Q_{r}$ também admite o modelo de fila $\mathrm{M} / \mathrm{M} / 1 / \mathrm{K}$.

Estas filas podem estar vazias, parcialmente ocupadas ou totalmente cheias (bloqueadas). As fórmulas para ambas as filas são similares.

Probabilidade de a fila $Q_{s}$ estar vazia:

$$
P_{s 0}(x)=\left\{\begin{array}{ll}
\frac{1-\rho_{s}(x)}{1-\rho_{s}(x)^{K+1}} & , \rho_{s}(x) \neq 1 \\
\frac{1}{K+1} & , \rho_{s}(x)=1
\end{array}\right\}
$$

Probabilidade de a fila $Q_{s}$ estar bloqueada:

$$
P_{b s}(x)=\left\{\begin{array}{ll}
\frac{\left[1-\rho_{s}(x)\right] \rho_{s}(x)^{k+1}}{1-\rho_{s}(x)^{k+1}} & , \rho_{s}(x) \neq 1 \\
\frac{1}{k+1} & , \rho_{s}(x)=1
\end{array}\right\}
$$

Fórmulas análogas valem para a fila $Q_{r}$ para o cálculo de $P_{r 0}(x)$ e $P_{b r}(x)$.

Para calcular as probabilidades das filas estarem vazias, $P_{s 0}(x)$ e $P_{r 0}(x)$, é preciso saber a intensidade de tráfego de cada uma, $\square_{s}(x)$ e $\square_{r}(x)$. Por sua vez, tais intensidades dependem desuas respectivas taxas de entrada:

$$
\begin{aligned}
& \rho_{s}(x)=\lambda_{s}(x) / \mu_{s}(x) \quad \mathrm{e} \\
& \rho_{r}(x)=\lambda_{r}(x) / \mu_{r}(x)
\end{aligned}
$$


A lei de conservação de fluxo permite determinar a relação entre as taxas de entrada em cada nó: o que sai do nó a $x+1$ saltos deve ser igual ao que entra na fila $Q_{r}$ do nó a $x$ saltos, ou seja,

$$
\begin{aligned}
& N(x+1) \sigma(x+1)=N(x) \lambda_{r}(x) \text { ou } \\
& \lambda_{r}(x)=N_{r}(x) \sigma(x+1)
\end{aligned}
$$

Substituindo (4), (3) e (7) em (8), chega-se a uma relação recursiva onde $\square_{r}(x)$ é definidoem termos de $\square_{r}(x+1)$ :

$$
\begin{array}{r}
\lambda_{r}(x)=N_{r}(x) \cdot\left[\sigma_{s}(x+1)+\sigma_{r}(x+1)\right]=N_{r}(x) \cdot \sigma_{s}(x+1)+N_{r}(x) \cdot \mu_{r}(x+1) \cdot\left[1-P_{r 0}(x+1)\right]= \\
N_{r}(x) \cdot \sigma_{s}(x+1)+N_{r}(x) \cdot \mu_{r}(x+1)-N_{r}(x) \cdot \mu_{r}(x+1) P_{r 0}(x+1)
\end{array}
$$

Nos nós mais afastados do gateway, a H saltos de distância, não há fila deencaminhamento, então, $\square_{r}(H)=0$ e $P_{r 0}(H)=1$.

Assim, se, na equação (9), $x=H-1$ :

$$
\begin{gathered}
\lambda_{r}(H-1)=N_{r}(H-1) \sigma_{s}(H)+N_{r}(H-1) \mu_{r}(H)-N_{r}(H-1) \mu_{r}(H) P_{r 0}(H) \text {, e portanto, } \\
\lambda_{r}(H-1)=N_{r}(H-1) \sigma_{s}(H) .
\end{gathered}
$$

$\square_{s}(H)$ é dado pela equação (3), conhecido a partir de $P_{s}(H)$ e $\square_{s}(H)$ das equações (5) e (7), que por sua vez são obtidos de $\square_{s}(H)$ e $\mu_{s}(H)$ que são parâmetros do sistema. Com o $\square_{r}(H-1)$ obtido calcula-se, analogamente, o próximo termo $\square_{r}(H-2)$. Assim sucessivamente até $\square_{r}(2), \square_{r}(1)$.

\subsection{CÁlCULO DA VAZÃO}

Define-se a vazão $V(x)$ como o número médio de pacotes entregue com sucesso ao destino por unidade de tempo, ou seja, a vazão de um nó a $x$ saltos é a taxa de pacotes que saiu desua própria fila $Q_{s}$ e não foi bloqueada em nós intermediários. Assim, para calcular a vazão é preciso conhecer a probabilidade de bloqueio na fila de origem do fluxo, $Q_{s}$, e as probabilidades de bloqueio nas filas de encaminhamento, $Q_{r}$, em cada nó intermediário. Se o nó for o vizinho do gateway, ou $x=1$, sua vazão será afetada apenas pelo bloqueio de sua fila local.

$$
V(x)=\left\{\begin{array}{cl}
\sigma_{s}(x)\left[1-P_{b s}(x)\right] & , x=1 \\
\sigma_{s}(x)\left[1-P_{b s}(x)\right] \prod_{i=1}^{x-1}\left[1-P_{b r}(i)\right] & , x=2 \ldots H
\end{array}\right\}
$$


Define-se vazão agregada como a soma da vazão de todos os nós:

$$
V_{\text {agg }}=\sum_{x=1}^{H}[N(x) \cdot V(x)]
$$

A vazão média por nó é dada por Vmed = Vagg/N.

\subsection{CÁlCULO DO ATRASO}

Para calcular o atraso fim-a-fim - o tempo gasto em todo o trajeto do pacote na malha desde a origem até o destino final - é preciso conhecer o atraso em cada fila que é proporcional aonúmero esperado de elementos na fila conhecido no estado estacionário. O número esperado de pacotes em cada fila, $L_{r}(x)$, é dado pelas fórmulas da fila M/M/1/K que podem ser vistas em (Gross e Harris, 1998):

$$
L_{r}(x)=\left\{\begin{array}{ll}
\frac{\rho_{r}(x)}{1-\rho_{r}(x)}-\frac{\rho_{r}(x)\left[K \rho_{r}(x)^{K}+1\right]}{1-\rho_{r}(x)^{K+1}} & , \rho_{r}(x) \neq 1 \\
\frac{K(K-1)}{2(K+1)} & , \rho_{r}(x)=1
\end{array}\right\}
$$

Fórmula análoga se aplica ao cálculo de $L_{s}(x)$, correspondente à fila $Q_{s}$.

$\mathrm{O}$ atraso relativo à espera na fila $Q_{r}$ é dado por

$$
W_{r q}(x)=\frac{L_{r^{\prime}}(x)}{\lambda_{r}(x) \cdot\left[1-P_{b r}(x)\right]}
$$

E o atraso total relativo à fila $Q_{r}$ é o atraso da espera na fila $Q_{r}$ mais o atraso do tempode serviço associado:

$$
W_{r}(x)=W_{r q}(x)+\frac{1}{\mu_{r}(x)}
$$

Note que $W_{r}(H)=0$. O atraso na fila $Q_{s}, W_{s}(x)$, é similar.

O atraso fim-a-fim é definido como o tempo entre a inserção do pacote na fila deorigem até o pacote ser totalmente recebido na MBS, portanto, é a soma dos tempos de enfileiramento e tempos de transmissão ao longo do caminho. O tempo de propagação é considerado desprezível. Assim o atraso fim-a-fim é a soma dos tempos na primeira fila $Q_{s}$, dos tempos nas filas intermediárias $Q_{r}$ e dos tempos 
de transmissão. A composição do atraso fim-a- fim está ilustrada na Figura 4. Considera-se que um pacote será transmitido em uma fatia de tempo $t_{c}$ e este é o tempo de transmissão considerado.

Figura 4 - Atraso fim-a-fim do nó a $x$ saltos até a MBS.

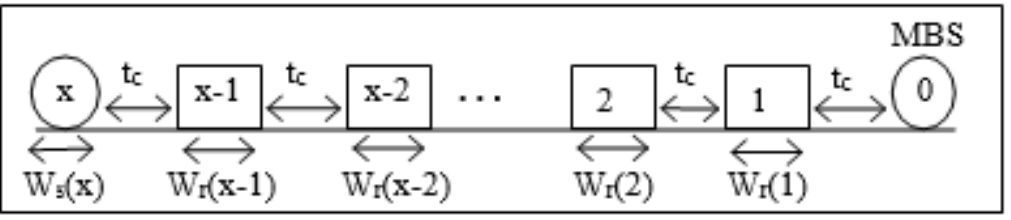

Decorre daí a fórmula do atraso :

$$
A(x)=\left\{\begin{array}{cc}
W_{s}(x)+t_{c}, & x=1 \\
W_{s}(x)+x t_{c}+\sum_{i=1}^{x-1} W_{r}(i), & x=2 \ldots H
\end{array}\right\}
$$

E o atraso médio em toda a malha é

$$
A_{\text {med }}=\frac{\sum_{x=l}^{H}[N(x) V(x) A(x)]}{\sum_{x=l}^{H}[N(x) V(x)]}
$$

\section{EQUIDADE}

Em diferentes cenários onde o modelo foi aplicado, variando as probabilidades $p(x), q(x) \mathrm{e}$ $\square_{s}(x)$, verificou-se que os nós mais distantes da MBS são extremamente prejudicados. A aplicação de um critério de equidade é fundamental para permitir um uso razoável de banda pelosnós mais distantes, pois em caso contrário, os nós vizinhos da MBS, como tem maior carga, para despachá-la, tenderiam a monopolizar a banda. $\mathrm{O}$ critério adotado neste artigo é dar a mesma banda ao nó $x$ que é dada a cada um dos nós para os quais $x$ é encaminhador.

Para fazer este cálculo é necessário contar quantos nós em média são servidos pelo nó a $x$ saltos da MBS. Define-se $R(x)$ como a soma do número de nós para os quais o nó a $x$ saltos serve. Se $x=H$, $R(x)=0$, pois o último nó não encaminha dados para nenhum outro nó.

Se $x=H-1$, ele serve os nós a H saltos, ou seja, $R(H-1)=N_{r}(H-1)$.

Se $x=H-2$, ele serve os nós a H-1 saltos diretamente e também os nós ligados aos nós H-1, ou seja, 
Aplicando este raciocínio sucessivamente chega-se a

$$
R(x)=\sum_{i=x}^{H-1} \prod_{j=x}^{i} N_{r}(j) \quad \text { ou } \quad R(x)=N_{r}(x)[1+R(x+1)]
$$

Conhecendo o conjunto dos nós servidos pelo nó a $x$ saltos, pode-se aplicar o critério de equidade que atribui à taxa de saída da fila $Q_{s}$, a mesma taxa da fila $Q_{r}$ repartida entre todos os nós servidos:

$$
\begin{aligned}
& \sigma_{s}(x)=\sigma_{r}(x) / R(x) \quad \text { ou } \\
& \sigma_{r}(x)=\sigma_{s}(x) \cdot R(x)
\end{aligned}
$$

Substituindo as equações (3), (2) na equação (17) chega-se a relação:

$$
q(x)\left[1-P_{r 0}(x)\right]=(1-q(x))\left[1-P_{s 0}(x)\right] R(x)
$$

Considerando as filas $Q_{s}$ e $Q_{r}$ em equilíbrio, tem-se $\operatorname{Ps}(\mathrm{x})<1$ e $\operatorname{Pr}(\mathrm{x})<1$. Assim na fórmula (5) da fila vazia assume-se que

$$
P_{s 0}(x)=\frac{1-\rho_{s}(x)}{1-\rho_{s}(x)^{K+1}}
$$

Considerando o tamanho máximo da fila, $\mathrm{K}$, suficientemente grande, vale aaproximação: $P_{s} 0(x)$ $=1-\square_{s}(x)$. Esta aproximação sendo considerada em (18) leva à seguinte relação

$$
\lambda_{r}(x)=\lambda_{s}(x) R(x)
$$

Dessa forma, para haver equidade é preciso haver esta proporcionalidade entre as taxas de chegada das duas filas no nó.

A probabilidade $p(x)$, embora na prática esteja vinculada a um controle de acesso ao meio específico, pode ser analisada, neste modelo abstrato, para verificar sua influência na melhoria da vazão e da equidade. A probabilidade $q(x)$ depende da política adotada para escolha da fila e também pode ser investigada na busca de um valor que auxilie a equidade na rede.

$\mathrm{O}$ ponto de partida da investigação é a suposição de que as filas estão em equilíbrio, ou seja, $\mathrm{P}_{S}$ $(x)<1$ e $\mathrm{P}_{r}(x)<1$. Começando com a fila $Q_{s}$ e usando as equações (1) e (2): 


$$
\rho_{s}(x)<1 \Rightarrow \frac{\lambda_{s}(x)}{\mu_{s}(x)}<1 \Rightarrow \frac{\lambda_{s}(x)}{\mu(x)(1-q(x))}<1 \Rightarrow \frac{\lambda_{s}(x) \cdot t_{c}}{p(x) \cdot(1-q(x))}<1 \Rightarrow 1-\frac{{\Lambda_{s}(x) \cdot t_{c}}_{p(x)}}{p q(x)}
$$

Esta condição de equilíbrio define, portanto um limite superior para $q(x)$. A fila $Q r$ também se considera em equilíbrio, então:

$$
\rho_{r}(x)<1 \Rightarrow \frac{\lambda_{r}(x)}{\mu_{r}(x)}<1 \Rightarrow \frac{\lambda_{r}(x)}{\mu(x) \cdot q(x)}<1 \Rightarrow \frac{\lambda_{r}(x) \cdot t_{C}}{p(x)}<q(x)
$$

Que representa um limite inferior para $q(x)$. Substituindo $\lambda_{r}(x)$ pela equação (19) olimite inferior para $q(x)$ em termos de $\lambda_{s}(x) e ́$

$$
\frac{\lambda_{s}(x) \cdot R(x) \cdot t_{c}}{p(x)} q a(x)
$$

Igualando os limites, inferior e superior, extrai-se $p(x)$ :

$$
1-\frac{\lambda_{s}(x) t_{c}}{p(x)}=\frac{\lambda_{s}(x) t_{c} R(x)}{p(x)} \rightarrow n(r)=\lambda_{s}(x) t_{c}(1+R(x))
$$

Nota-se que a probabilidade $p(x)$ justa é diretamente proporcional à taxa de chegada da fila $Q_{s}$ do nó e sua localização na topologia da rede. Quanto mais nós o nó a $x$ saltos serve, maior deve ser sua chance de transmitir. Porém o modelo equilibra o favorecimento aos nós mais ativos com o favorecimento dos mais distantes que na ausência deste critério sofreriam de inanição.

Como não se está considerando o reúso espacial, e apenas um nó por vez ganharáacesso ao meio, a soma das probabilidades dos nós deve ser igual a 1.

$$
\sum_{x=1}^{H} N(x) p(x)=1
$$

Uma possibilidade de solução para determinação de $p(x)$ seria resolver o sistema de equações (22) e (23) para determinadas taxas $\square_{s}(x)$ de entrada. Verificou-se em diversos cenários que nem sempre este sistema de equações tem solução para quaisquer taxas de entrada. Em contrapartida é possível encontrar uma taxa de entrada justa compatível com este sistema de equações. 
Para chegar à taxa de entrada justa, nota-se que, como consequência da aplicação do critério de justiça, as taxas de entrada de todos os nós devem ser iguais. Isto pode ser observado desenvolvendo a equação (19) :

$$
\begin{aligned}
\Rightarrow \quad \lambda_{r}(x)=\lambda_{s}(x) R(x) & =>N_{r}(x) \sigma(x+1)=\lambda_{s}(x) R(x)=>\sigma_{s}(x+1)+\sigma_{r}(x+1)=\lambda_{s}(x) R(x) / N_{r}(x) \\
& \mu_{s}(x+1)\left[1-P_{s 0}(x+1)\right]+\mu_{r}(x+1)\left[1-P_{r 0}(x+1)\right]=\lambda_{s}(x) R(x) / N_{r}(x)
\end{aligned}
$$

Considerando as filas em equilíbrio e $\mathrm{K}$ grande pode-se substituir na fórmula (24) $P_{s 0}(x+1)$ e $P_{r 0}(x+1)$ por $1-\mathrm{P}_{s}(x+1)$ e $1-\mathrm{P}_{r}(x+1)$ respectivamente:

$$
\begin{aligned}
& \mu_{s}(x+1) \cdot \rho_{s}(x+1)+\mu_{r}(x+1) \cdot \rho_{r}(x+1)=\lambda_{s}(x) R(x) / N_{r}(x)=> \\
& \lambda_{s}(x+1)+\lambda_{r}(x+1)=\lambda_{s}(x) \cdot R(x) / N_{r}(x)=> \\
& \quad \lambda_{s}(x+1)+\lambda_{s}(x+1) R(x+1)=\lambda_{s}(x) R(x) / N_{r}(x)=>\lambda_{s}(x+1) \\
& \lambda_{s}(x)
\end{aligned}
$$

Mas, de (16) sabe-se que $\mathrm{R}(\mathrm{x})=\mathrm{Nr}(\mathrm{x})[1+\mathrm{R}(\mathrm{x}+1)]$, e, portanto $\square \mathrm{s}(\mathrm{x}+1) / \square \mathrm{s}(\mathrm{x})=1$, o que significa que as taxas de entrada justas nos diversos nós são necessariamente iguais, ou seja, $\lambda s(\mathrm{x})=\lambda \mathrm{s}$.

Substituindo (22) em (23) chega-se à taxa de entrada justa que deve se restringir em cada nó:

$$
\sum_{x=1}^{H} N(x) \cdot \lambda_{s^{\circ} c}[1+R(x)]=1 \Rightarrow \lambda_{r}=\frac{1}{\sum_{x=1} N(x) t_{c}[1+R(x)]}
$$

Com esta taxa restritiva, calcula-se a probabilidade $p(x)$ pela fórmula (22) e a seguir aplica-se $p(x)$ à equação (21) para determinar a probabilidade $q(x)$ justa para a escolha das filas.

\section{ANÁLISE DE RESULTADOS}

A primeira validação obtida refere-se a comparação entre o modelo proposto e omodelo de Liu e Liao (2008), onde os autores assumem que a fila $Q_{s}$ é acumulada (backlogged), ou seja, nunca está vazia, nem excessivamente cheia e, portanto, não é do tipo $M / M / 1 / K$. As

Figuras 5(a) e 5(b) mostram a coincidência da implementação de (Liu e Liao, 2008) com o modelo proposto, ambos aplicados ao mesmo cenário. No teste onde se buscava a coincidência foram variados os parâmetros relativos à fila e uma boa aproximação foi encontrada com os parâmetros: Tamanho da Fila, $K_{s}=30$ e Taxa de entrada na Fila Local, $\lambda_{s}=800$ pacotes $/$ segundo. 
Na Figura 5(a) é apresentada a comparação da variação da vazão em função da distância a MBS e em 5(b) a variação do atraso. Isto quer dizer que os resultados do modelo de Liu e Liao (2008) representam um caso particular em certa configuração específica, e que o modelo aqui apresentado é mais geral.

Figura 5 - Comparação dos Modelos

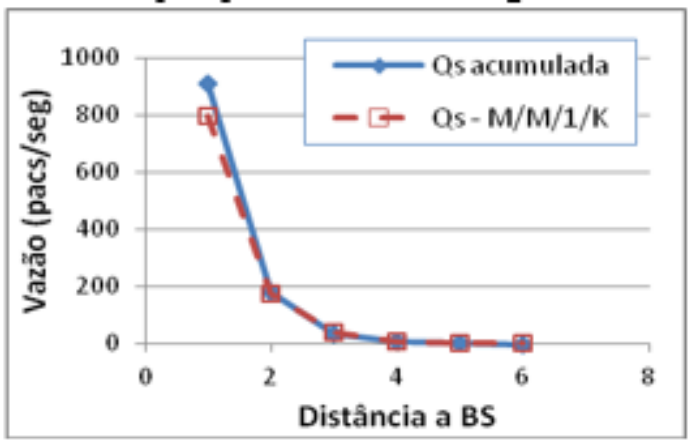

(a) Vazão

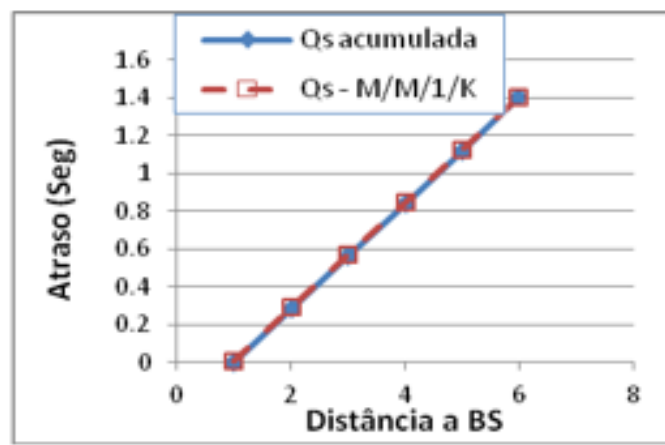

(b) Atraso

Visando a análise de desempenho em função das probabilidades $p(x)$ e $q(x)$ estasprobabilidades foram variadas em diferentes topologias. Serão apresentadas a seguir duas diferentes topologias que ilustram a avaliação dos resultados. O primeiro cenário assume uma topologia em cadeia, como na Figura 6 onde cada nó tem apenas dois vizinhos e a MBS é o nó derótulo zero.

O segundo cenário, na Figura 7, assume a topologia em grade onde um nó no interior dagrade tem quatro vizinhos diretos e a MBS é o nó de rótulo vinte e quatro. Em todos os testes foi utilizada a taxa de entrada $\lambda_{s}$ igual em todos os nós: Com esta premissa, nos dois cenários foram realizados três tipos de testes:

(1) Aplicação de probabilidades de acesso ao meio $p(x)$ iguais para todos os nós: $p(1)=p(2) \ldots p(H)=1 /(N-1)$ e probabilidade de escolha da fila $Q_{r}$ baixa: $q(x)=0.2$, teste denominado $\mathbf{q}=\mathbf{0 . 2}$ nos gráficos;

(2) Aplicação de probabilidades de acesso ao meio $p(x)$ iguais para todos os nós: $p(1)=p(2) \ldots p(H)=1 /(N-1)$ e probabilidade de escolha da fila $Q_{r}$ alta: $q(x)=0.8$, teste denominado $\mathbf{q}=\mathbf{0 . 8}$ nos gráficos;

(3) Aplicação de $p(x)$ da equação (22) e $q(x)$ da equação (21), teste denominado Fair nos gráficos.

Uma medida de comparação importante do desempenho é a medida do critério de justiça, ou seja, do quanto a distribuição dos recursos foi equânime. Um índice facilmente calculável e de larga utilização na área é o índice de Jain (1984). Se $v_{i}$ é a vazão de saída do nó $i$, e $N$ é o número de nós que compartilham o recurso, o índice de Jain é definido como: 
Figura 6-Topologia em cadeia

$$
I=\frac{\left[\sum_{i=1}^{N} v_{i}\right]^{2}}{N \sum_{i=1}^{N} y^{2}}
$$

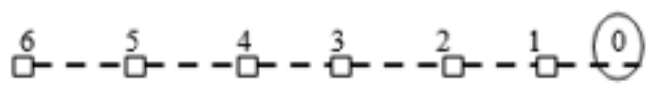

Figura 7 - Topologia em grade

\begin{tabular}{|lllllll|}
\hline 0 & 1 & 2 & 3 & 4 & 5 & 6 \\
7 & 8 & 9 & 10 & 11 & 12 & 13 \\
14 & 15 & 16 & 17 & 18 & 19 & 20 \\
21 & 22 & 23 & 24 & 25 & 26 & 27 \\
28 & 29 & 30 & 31 & 32 & 33 & 34 \\
35 & 36 & 37 & 38 & 39 & 40 & 41 \\
42 & 43 & 44 & 45 & 46 & 47 & 48 \\
\hline
\end{tabular}

\section{(A) Topologia Cadeia}

O teste realizado na topologia cadeia considera a distância máxima de 6 saltos da MBS, ou seja, $\mathrm{H}=6$.

Nesta topologia $N(x)=1$, qualquer que seja o $x$. O gráfico da Figura 8 indica grande ganho na vazão de cada nó com a abordagem justa.

No caso de $\mathbf{q}=\mathbf{0 . 2}$, o nó vizinho da MBS dá preferência aos próprios dados e, portanto atinge alta vazão para si, em detrimento do encaminhamento o que causa uma queda vertiginosa da vazão dos demais nós da cadeia. Já no caso $\mathbf{q = 0 . 8}$, ao dar prioridade ao encaminhamento, o desempenho tem significativa melhora, porém o nó mais distante é beneficiado, como se todos osoutros nós trabalhassem mais para ele.

A Figura 9 ilustra a variação do atraso. Não houve intenção explícita no modelo de melhoria do tempo, pois o critério de justiça refere-se à vazão, mas nota-se da figura que a abordagem justa também traz melhorias em relação ao atraso.

Figura 8 -Vazão na topologia cadeia

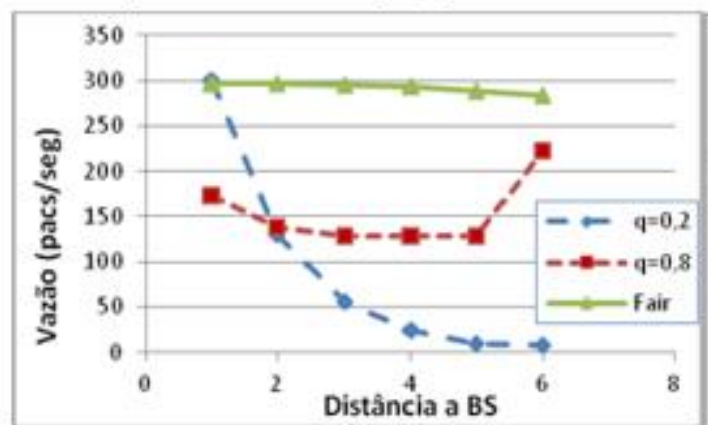

Figura 9 -Atraso na topologia cadeia

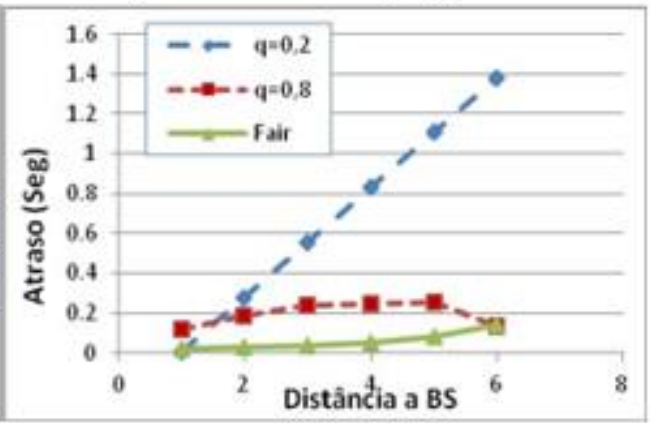

No teste Fair, variando $p(x)$ e $q(x)$ adequadamente, consegue-se um equilíbrio entre encaminhar os próprios dados e os dados dos vizinhos, ocasionando uma melhoria na vazão de todos os nós. O índice de Jain $J$ medido para os 3 casos foi: 
- $\quad \mathbf{q}=\mathbf{0 . 2}: J=0.42$;

- $\quad \mathbf{q}=\mathbf{0 . 8}: J=0.95$;

- $\quad$ Fair: $J=0.99$;

(B) Topologia Grade

A topologia em grade ilustrada na Figura 7 também considera a distância máxima

H =6 saltos da MBS. Embora a distância máxima seja a mesma do teste feito na topologia em cadeia, neste caso a densidade é maior, ou seja, há mais nós à mesma distância e mais nós com a missão do encaminhamento e, portanto mais competição, o que fez a vazão obtida ser bem menor. Nesta topologia $N(x)$ varia e também $N_{r}(x)$.

O gráfico da Figura 10 também indica ganho na vazão de cada nó com a abordagem justa, de maneira similar a topologia cadeia. Também no caso do atraso o gráfico da Figura 11 indica o ganho na abordagem justa na topologia em grade de maneira similar a topologia cadeia.

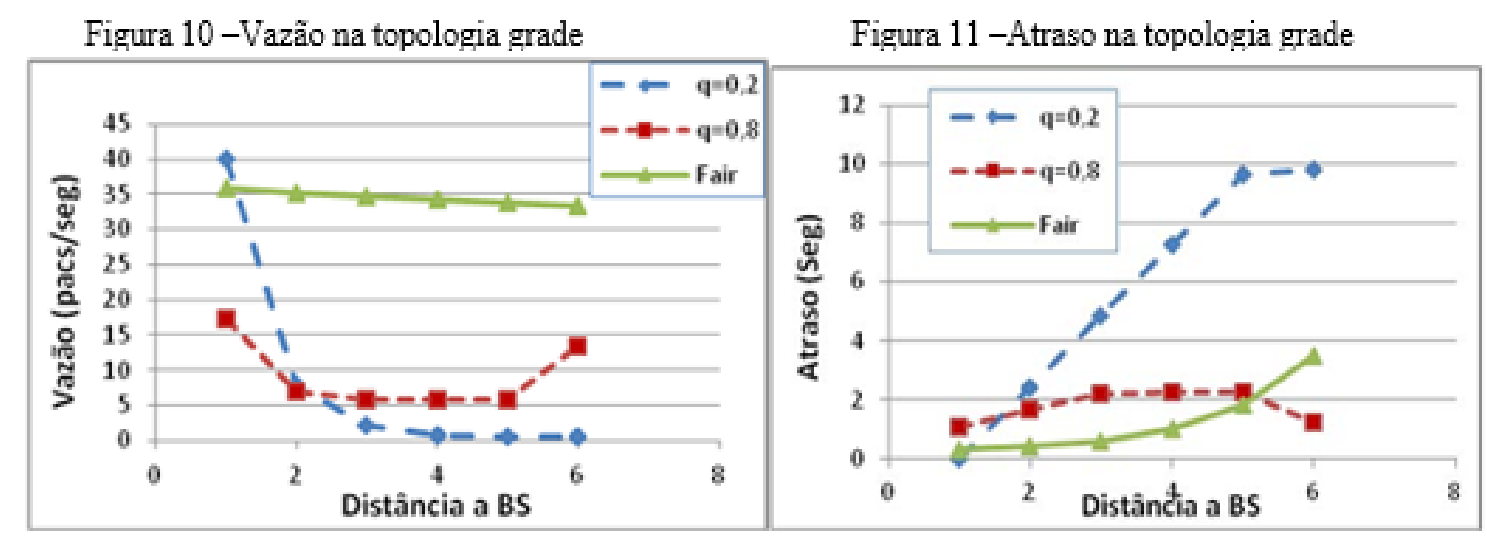

A Figura 12 ilustra a soma da vazão média em cada nó da rede comparando os três tiposde teste. Esta figura enfatiza que a busca da justiça na rede causou um ganho de vazão da ordem de $80 \%$ na rede como um todo, em comparação com o segundo melhor caso, $\mathrm{q}=0.8$.

Na Figura 13 temos a soma da vazão em todos os nós na topologia em grade que é muito superior quando se aplica o critério de justiça. 
Figura 12:Vazão agregada na topol. Cadeia

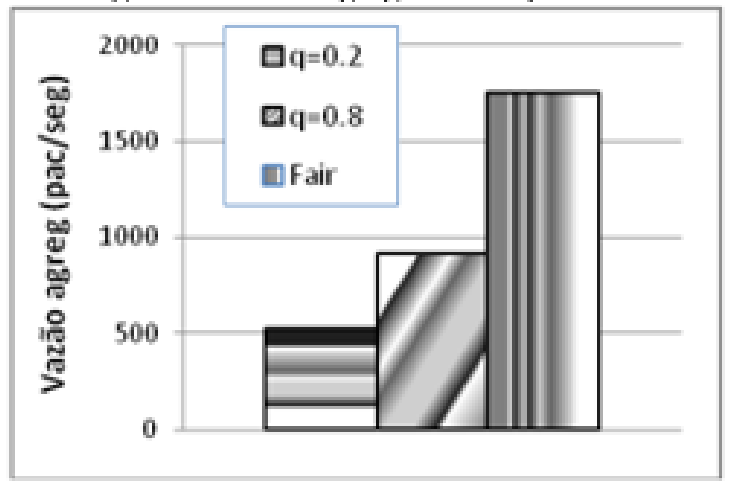

Figura 13: Vazão agregada na topol. Grade

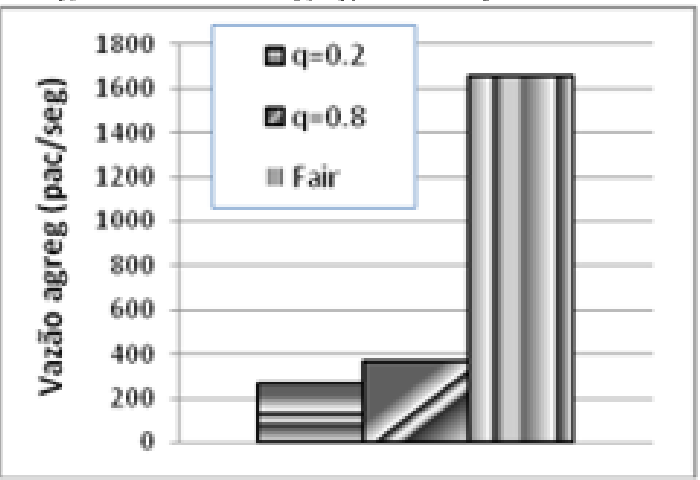

Finalmente, nas Figuras 14 e 15 encontram-se as variações das probabilidades $p(x)$ e $q(x)$ na abordagem justa em relação as demais abordagens. Nota-se da Figura 14 que o ganho no desempenho verificado nos gráficos anteriores deve-se ao controlado favorecimento dos nós próximos a MBS, uma vez que como tem maior volume de dados, justifica-se que tenham maior acesso ao meio. Em relação à escolha da fila Qr, verificou-se que também os nós mais próximos da MBS devem privilegiar o encaminhamento, e esta priorização vai se tornando menos importante com a distância a MBS, pois há um volume menor de dados a encaminhar.

Figura 14 - Variação de $p(x)$

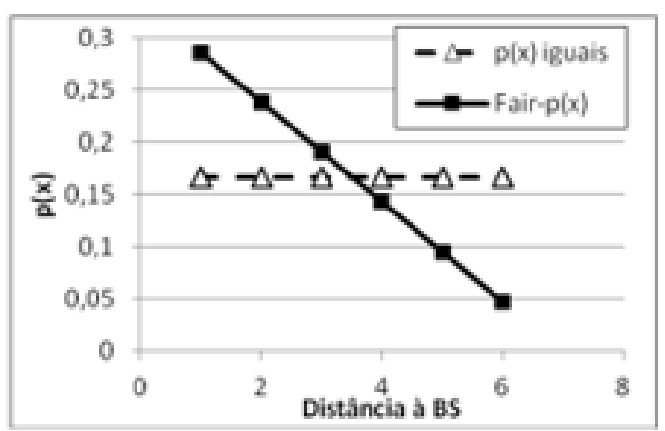

Figura 15 - Variação de $q(x)$

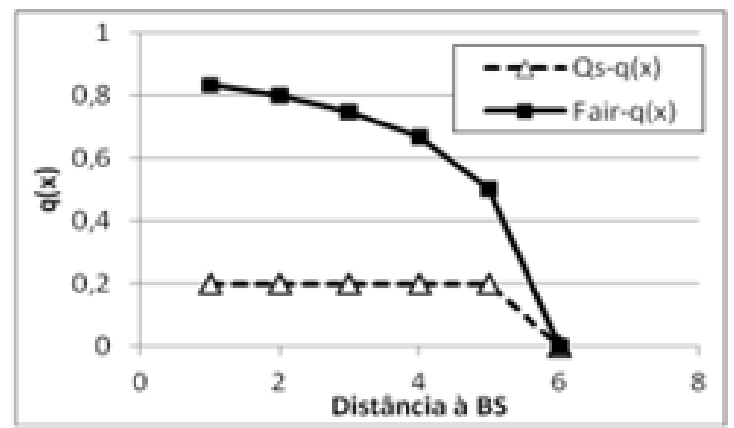

\section{CONCLUSÃO}

Este artigo apresentou um modelo analítico usando Teoria de Filas para cálculo devazão e atraso em uma rede de múltiplos saltos. A modelagem apoia-se em duas probabilidades:

$p(x)$, probabilidade de um nó a $x$ saltos da MBS ganhar acesso ao meio; e $q(x)$, probabilidade de escolher pacotes para transmissão da fila de encaminhamento dos nós vizinhos.

Foi feita uma comparação com os resultados do modelo de Liu e Liao (2008) e verificou-se que, em uma específica configuração, os modelos coincidem. Isto enfatiza que o modelo proposto aqui é mais abrangente, porque permite variar o tamanho da fila local e sua taxade entrada, além de tratar os casos extremos de fila local cheia ou vazia. 
Um critério de equidade foi apresentado para calcular as probabilidades $p(x)$ e $q(x)$ visando a uma distribuição justa da banda disponível. A equidade em si foi também mensurada, atingindo, numa escala entre 0 e 1 , o índice 0.99 . Os resultados indicam que, quando se restringea taxa de entrada de cada nó a uma fração justa, e nos diversos nós se equilibram as probabilidades $p(x)$ e $q(x)$, os ganhos são significativos, podendo chegar a $80 \%$ da vazão em determinados cenários. 


\section{REFERÊNCIAS}

Akyildiz, I. F.; Wang, X.; Wang,W. "Wireless mesh networks: a survey". Computer Networks Journal (Elsevier). p. 445-487, January, 2005.

César, C. A. C. "Um modelo analítico para análise do desempenho de redes mesh IEEE 802.16 com escalonamento distribuído", 2011. 167 p. (sid.inpe.br/mtc-m19/2011/05.31.15.16- TDI). Tese (Doutorado em Computação Aplicada) - INPE, São José dos Campos, 2011. Disponível em: <http://urlib.net/8JMKD3MGP7W/39Q5AEH>. Acesso em: 10 abr. 2012.

Duffy, K.; Leith, D. J.; Li, Malone, T. D. "Improving fairness in multi-hop mesh networks using 802.11e". Modeling and Optimization in Mobile, Ad Hoc and Wireless Networks, 2006 4th International Symposium on , pp. 1- 8, 03-06 April 2006.

Gambiroza, V.; Sadeghi, B.; Knightly, E. W. "End-to-end Performance and Fairness in Multihop Wireless Backhaul Networks". In Proc. International Conference on MobileComputing and Networking. 2004, Philadelphia. New York: ACM, 2004. p. 287-301.

Gross, D.; Harris, C. “Fundamentals of Queueing Theory”, $3^{\text {rd }}$ ed. Hoboken,NJ: Wiley, 1998, pp. 74-80.

Gupta, P.; Kumar, P.R. "The capacity of wireless networks". IEEE Trans. Inf. Theory, vol 46, no2, pp.388-404. Mar, 2000.

Institute of Electrical and Electronic Engineers. IEEE 802.16-2004: IEEE Standard for Local and Metropolitan Area Networks - Part 16: Air Interface for Fixed Broadband Wireless Access Systems. Disponível em http://standards.ieee.org/getieee802/download/802.16-2004.pdf.

Jain, R.; Chiu;C.; Hawe, W. "A quantitative measure of fairness and discrimination for resource allocation in shared computer system”. Hudson: Digital Equipment Corp., 1984 40p.

$$
\text { Report, DEC-TR-301). Disponível }
$$

(Technical

http://www.cse.wustl.edu/ jain/papers/fairness.html. Acesso em 04 de abril de 2012.

Kuo, J.C;, Liao, W. "Hop Count Distribution of Multihop Paths in Wireless Networks with Arbitrary Node Density: Modeling and its applications”. IEEE Trans. Veh. Technol., vol 56, no 4, pp. 2321-2331, Jul. 2007.

Liu, B.; Liu, Z.; Towsley, D. "On the capacity of hybrid wireless networks". In Proc. IEEE INFOCOM, Mar. 2003, pp. 1543-1563.

Liu, T.; Liao, W.“Location-Dependent Throughput and Delay in Wireless Mesh Networks”, IEEE Transactions on Vehicular Technology, vol. 57, No 2, 07.pg 1188-1198, March2008.

Min, G.; Wu,Yy.; Li, K.; Ai-Dubai, A. Y. "Performance modelling and optimization of integrated wireless LANs and multi-hop mesh networks". International Journal of Communication Systems, v. 23, n. 9-10, p.1111-1126, 2009.

Wang, P.; JIANG, H.; Zhuang, W.; Poor, H.V. "Redefinition of max-min fairness in multi- hop wireless networks," Wireless Communications, IEEE Transactions on , vol.7, no.12, pp.4786-4791, December 2008.

Zhang, Y., Luo J., Hu H. "Wireless Mesh Networking - Architectures, Protocols and Standards". First Edition, Florida, EUA. Auerbach Publications, December 2006, p.610. 